\title{
A Galactic Center Excess in the Andromeda Galaxy M31 Seen with the Fermi-LAT
}

\author{
Xian Hou* \\ Yunnan Observatories, Chinese Academy of Sciences, 396 Yangfangwang, Guandu District, \\ Kunming 650216, P. R. China \\ Key Laboratory for the Structure and Evolution of Celestial Objects, Chinese Academy of \\ Sciences, 396 Yangfangwang, Guandu District, Kunming 650216, P. R. China \\ Center for Astronomical Mega-Science, Chinese Academy of Sciences, 20A Datun Road, \\ Chaoyang District, Beijing 100012, P. R. China \\ E-mail: xianhou.astrolgmail.com
}

\section{Pierrick Martin}

CNRS, IRAP, F-31028 Toulouse cedex 4, France

E-mail: pierrick.martin@irap.omp.eu

\section{On behalf of the Fermi LAT Collaboration}

The Fermi Large Area Telescope (LAT) has opened the way for comparative studies of cosmic-ray populations and high-energy sources in the Milky Way (MW) and in other external star-forming galaxies. Using more than seven years of LAT Pass 8 data in the energy range $0.1-100 \mathrm{GeV}$, M31 is detected at nearly $10 \sigma$ and is observed to be extended at $4 \sigma$. Its spectrum is consistent with a power law and its spatial distribution is consistent with a uniform brightness disk over the plane of the sky and no offset from the center of M31. The emission appears confined to the inner regions of the galaxy and does not fill the disk of the galaxy. The non-correlation with regions rich in gas or star-formation activity suggests that the emission is not interstellar in origin, unless the energetic particles radiating in gamma rays do not originate in recent star formation. Alternative interpretations include a population of unresolved millisecond pulsars in the galaxy center or dark matter annihilation or decay, similar to what has been proposed to account for the Galactic center excess found in LAT observations of the MW.

7th Fermi Symposium 2017

15-20 October 2017

Garmisch-Partenkirchen, Germany

\footnotetext{
* Speaker.
} 